\title{
An Adaptive Lyapunovs Internal PID Regulator in Automotive Applications
}

\author{
Paolo Mercorelli \\ Institute of Product and Process Innovation, \\ Leuphana University of Lueneburg, Volgershall 1, \\ D-21339 Lueneburg, Germany \\ mercorelli@uni.leuphana.de
}

\author{
Nils Werner \\ Faculty of Automotive Engineering, \\ Ostfalia University of Applied Sciences, \\ Kleiststr. 14-16, D-38440 Wolfsburg, Germany \\ n.werner@ostfalia.de
}

Received: June 14, 2019. Revised: September 26, 2021. Accepted: October 17, 2021. Published: November 23, 2021.

\begin{abstract}
This paper considers an aggregate actuator consisting of a piezo and a hydraulic part. Moreover a cascade PI-PID controllers is taken into account for its control in automotive applications. In the presented work the Preisach dynamic model is taken into consideration a long with a cascade PI-PID controllers. In particular, the hysteresis effect is considered and a model with a switching function is used also for the controller design. Simulations with real data are able to be seen in this contribution.
\end{abstract}

Key-words: PID controllers, Lyapunov's approach, piezo actuators

\section{INTRODUCTION}

Recently, a lot of interest has been paid to variable engine valve control because it can make smaller the pumping losses and to improve torque performance. Variable valve timing permits the control of internal exhaust gas recirculation, so making fuel economy better and making NOx emissions less.

This paper is in progress with the works [1], [2] and in Fig. 1 shows the phase diagram of the positions of an engine intake and exhaust valves. In this figure the intake and the exhaust valve position profile are indicated. Figure 1 demonstrates the new engine structure with, evidently, four piezo actuator. Generally speaking, this project closely concerns a new conception of the functionality of some parts of the engine, for example of the throttle valve control, see [3]. In spite of this, the inaccuracy in positioning control comes from the hysteresis effect. In this paper the hysteresis effect is model making use of a linearisation. A linear boundary of the hysteresis is taken into account and a switching approach is applied to follow the hysteresis characteristics. The easiest thought is to consider the upper and the lower bound of the linear characteristic. PID regulators are very often applied in industry. Recently, variable engine valve control has attracted a lot of interest because of its skill to make fuel economy better, make less NOx emissions and to increase torque performance over a wider rage than a conventional spark-ignition engine. Combined with microprocessor control, very important functions of the motor management can be well controlled by such mechatronic actuators. For moving distances between 5 and $8 \mathrm{~mm}$, however, there are other actuator kinds with different benefits. In [4] we demonstrated an adaptive PID controller design for the valve actuator control based on flatness property and interval polynomials. The
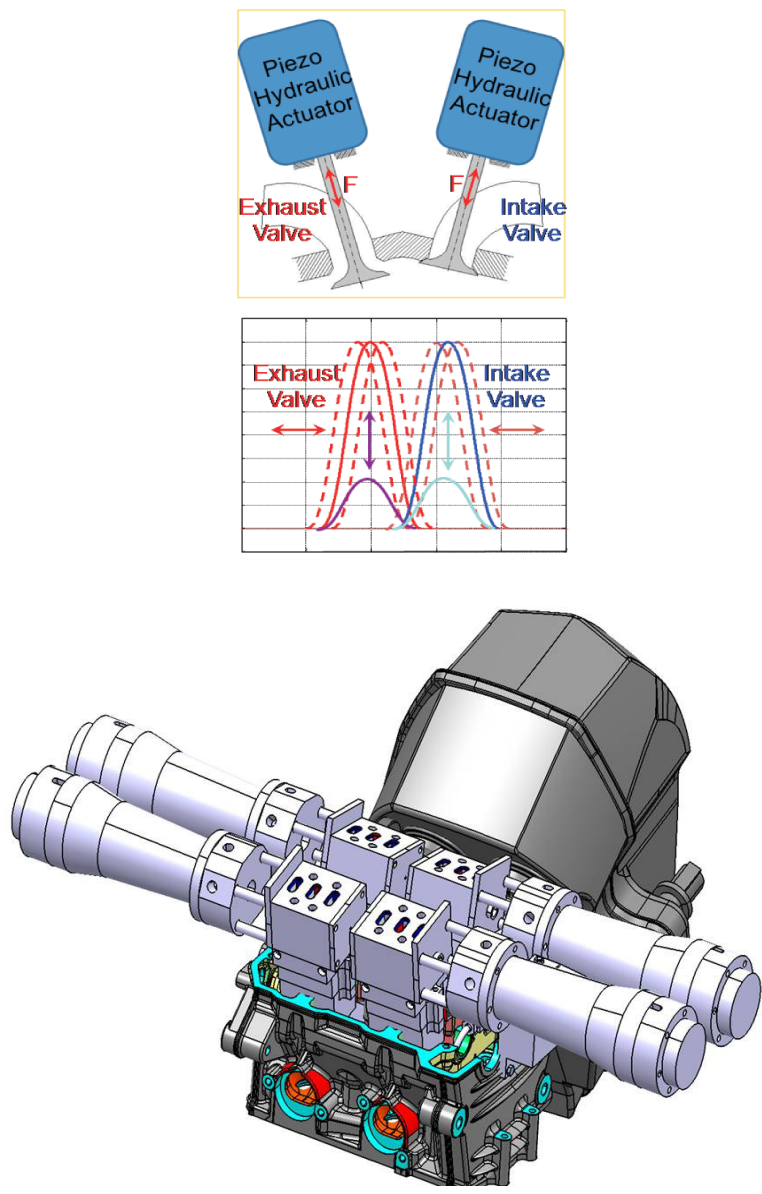

Fig. 1. New structure of the engine.

aim of this paper is showing:

- A model of the a hybrid actuator

- A PI-PID cascade regulator

A combination of a switching cascade PI-PID structure and feedforward regulators is demonstrated in this paper. The control structure is a switching one which considers the suggested switching model of the hysteresis effect.

The paper is divided into the following sections. Section II is dedicated the description of the model. In Section III an algorithm deriving the control laws is shown. The paper finishes with Section IV in which simulation results of the suggested valve using real data are presented. After that the conclusions can be found.

\section{The Model of the Hybrid Actuator}

Figure 3 shows the connections of the valves with the tank and with the pump. If connections T-A and P-B are open the 


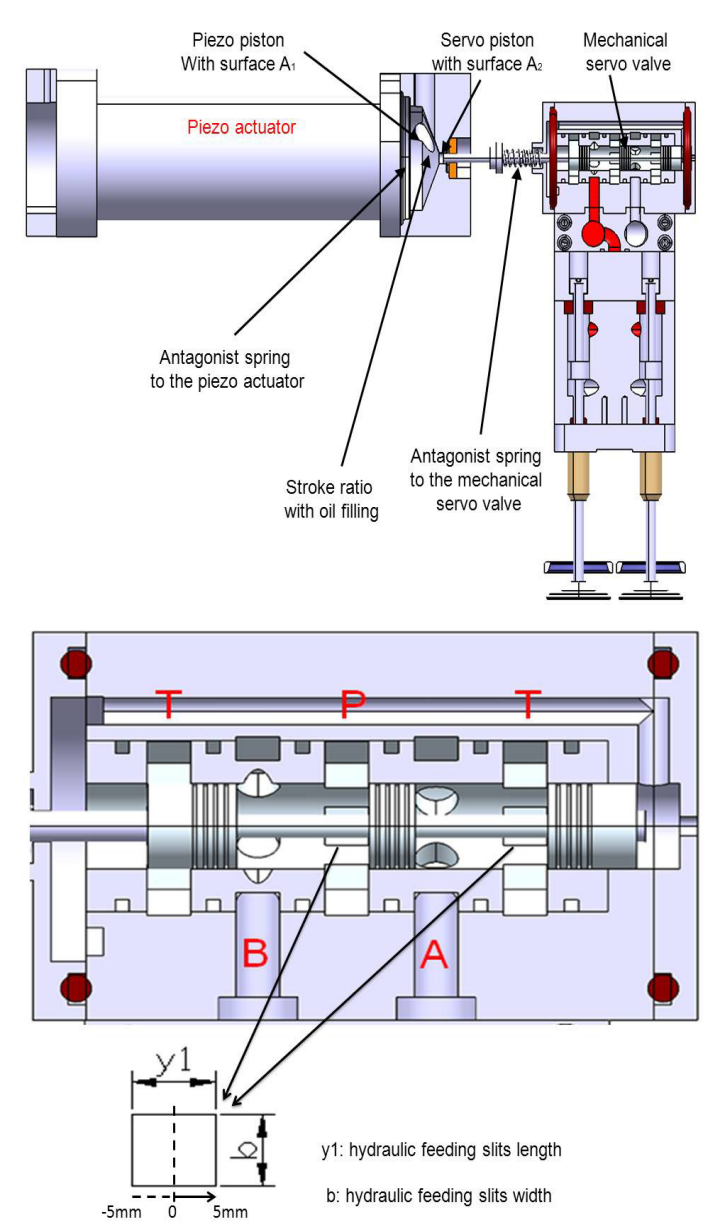

Fig. 2. Structure of the hybrid actuator and detail of the slits

valves are closed because the point $\mathrm{B}$ is under pressure. If the mechanical servo valve is in the middle position, both connections (T-A and P-B) are closed and connections A-P and B-T start opening and in this case both engine valves start opening because the point $\mathrm{A}$ is under pressure. Figure

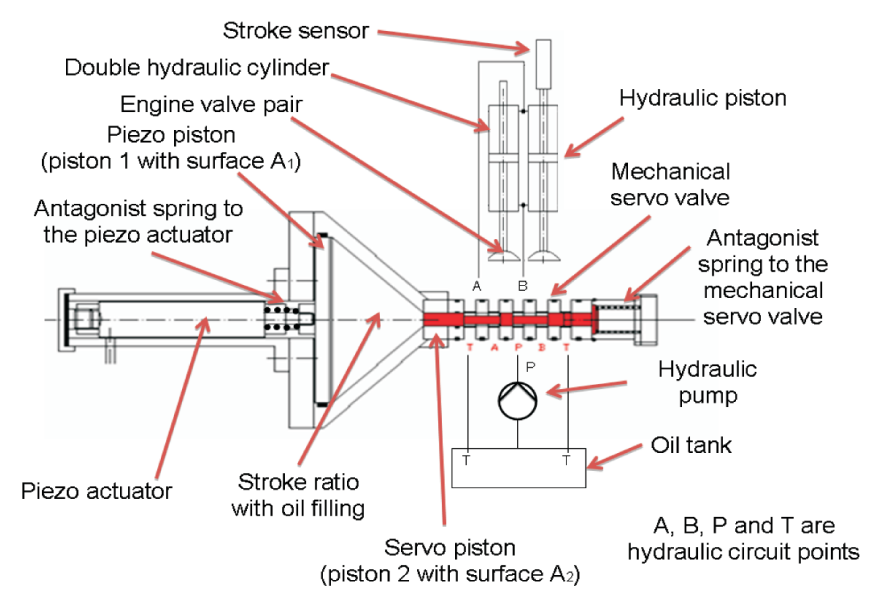

Fig. 3. Hybrid Piezo Hydraulic Actuator

2 fully describes a part of the hybrid actuator containing of

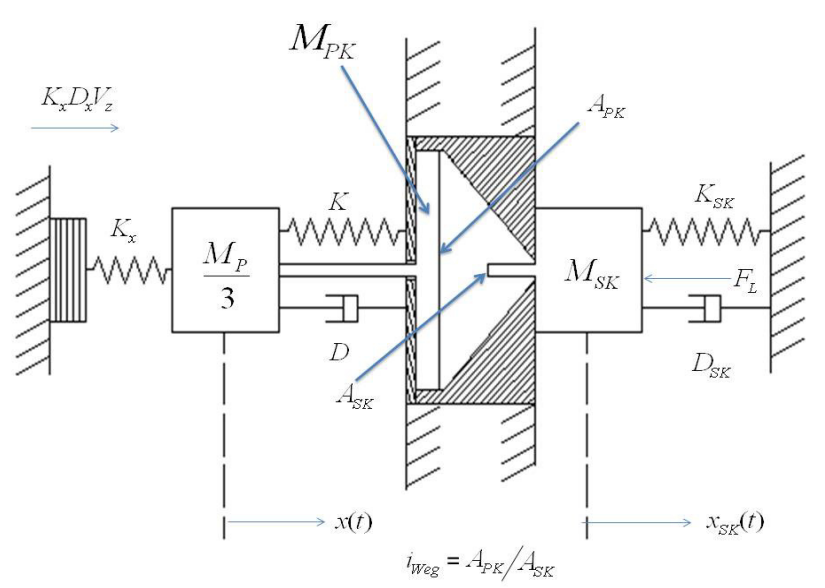

Fig. 4. Particular of the adapting stroke ration.

a piezo actuator combined with a mechanical part. The both parts are connected by a stroke ratio for adapting the stroke length. In 4 a detail of the mechanical stroke adapter can be found. The suggested structure of the model of the PEA is rather alike as those which can be seen in [5] and in [6]. The model consists of a sandwich structure as it can be seen in Fig. 5, being based on the hypothesis to be mentioned now.

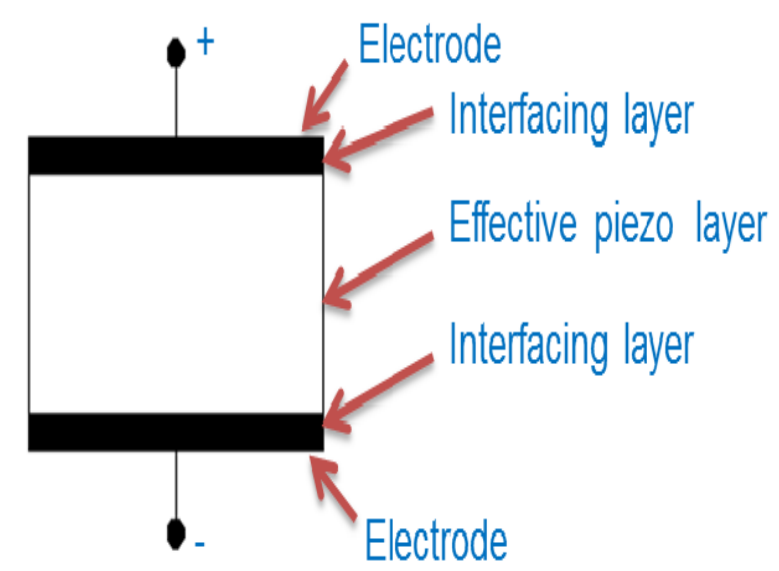

Fig. 5. The sandwich model of the PEA

Figure 6 shows the equivalent circuitry for a PEA, in which two I-layers are combined together as $C_{a}$ and $R_{a}$. The I-layer capacitor, $C_{a}$, is a common capacitor which is assumed to be constant. The I-layer resistor, $R_{a}$, consists of a nonlinear function. This resistance, $R_{a}>10^{6} \Omega$, when the voltage $\left\|V_{a}\right\|<V_{h}$, or is small, say $R_{a}<1000$, when $\left\|V_{a}\right\|>V_{h}$. In [6], voltage $V_{h}$ is defined as the hysteresis voltage of a PEA. The authors in [6] proposed a possible definition after observing that there is an important difference and a change in the resistance value. This change across $V_{h}$ that models the nonlinearities of hysteresis and creep effect in a piezo actuator. The hysteresis effect could be described as input $V_{i n}(t)$ and output $y(t)$ in this way: $H\left(y(t), V_{i n}(t)\right)$, see Fig. 7. If $V_{h}=0$, then the hysteresis effect goes away, and if 


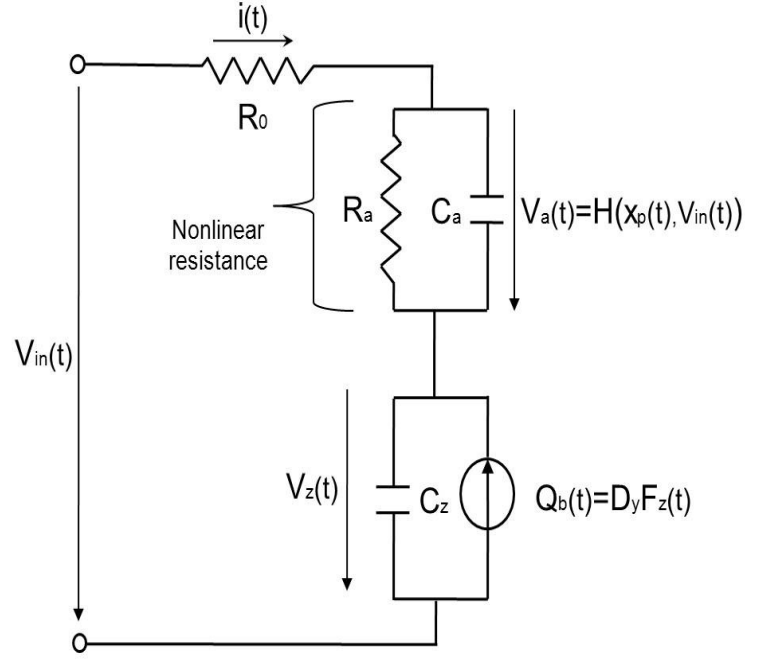

Fig. 6. Electrical part of the model

$R_{a}=\infty$ when $\left\|V_{a}\right\|<V_{h}$, in this case the creep effect goes away. Considering the electrical circuit as indicated in Fig. 6 , the state model is derived as follows:

$$
\begin{aligned}
& \dot{V}_{a}(t)=-\left(\frac{1}{R_{a}}+\frac{1}{R_{o}}\right) \frac{V_{a}(t)}{C_{a}}-\frac{V_{z}(t)}{C_{a} R_{o}}+\frac{V_{i n}(t)}{C_{a} R_{o}} \\
& \dot{V}_{z}(t)=\frac{\dot{Q}_{b}}{C_{z}}+\frac{1}{C_{z}}\left(-\frac{V_{a}(t)}{R_{o}}-\frac{V_{z}(t)}{R_{o}}+\frac{V_{i n}(t)}{R_{o}}\right),
\end{aligned}
$$

where $Q_{b}=D_{y} F_{z}(t)$ is the well known back electric charge force (back-ecf), as explained in [6]. Considering [6] and the representation in Fig. 8, it can be written as

$$
F_{z}(t)=M_{p} / 3 \ddot{x}(t)+D \dot{x}(t)+K x(t)+K_{x} x(t) .
$$

$K$ is the elasticity constant and $D$ represents the friction parameter of the spring. $C_{z}$ is the capacity of the piezo actuator and $R_{o}$ is the resistive effect of the contacts. A more detailed description of the model can be seen in [6]. If the model represented in Fig. 8 a long with the assumption that the oil is incompressible, then a possible mechanical model can be described using spring as it is indicated in Fig. 8 in which $K_{x}$ represents the elasticity effect of the piezo actuator. Factor $D_{x} K_{x}=T_{e m}$ is the "transformer ratio" and it indicates a fundamental property of the PEA. $M_{p} / 3$ represents the moving mass of the PEA and this is always a part of the total piezo mass, $M_{S K}$ is the sum of the following masses: piston mass, oil and the moving actuator masses. $M_{v}$ represents the valve mass. It can be noticed that the moving mass of the piezo structure is just a fraction of the complete piezo mass. The value of this fraction is given by the constructer of the piezo device and experimental measurements determine it.

$K_{S K}$ and $D_{S K}$ are constat which are associated to the spring of the mechanical servo piston, see Fig. 8. $D_{\text {oil }}$ represents the constant which are associated to the oil friction effect. Considering [6], the state variable $x_{p}(t)$ indicated in
Fig. 7 is

$$
x_{p}(t)=D_{x} V_{z}(t)
$$

From diagram of Fig. 6, the following expression can be derived:

$$
V_{z}=V_{i n}(t)-R_{0} i(t)-H\left(x_{p}(t), V_{i n}(t)\right),
$$

where $R_{0}$ resistance of the contacts and $i(t)$ is the current drawing through the PEA as indicated in Fig. 6 . $H\left(x_{p}(t), V_{i n}(t)\right)$ is the function describing the hysteresis effect and demonstrated in Fig. 7. If the complete system

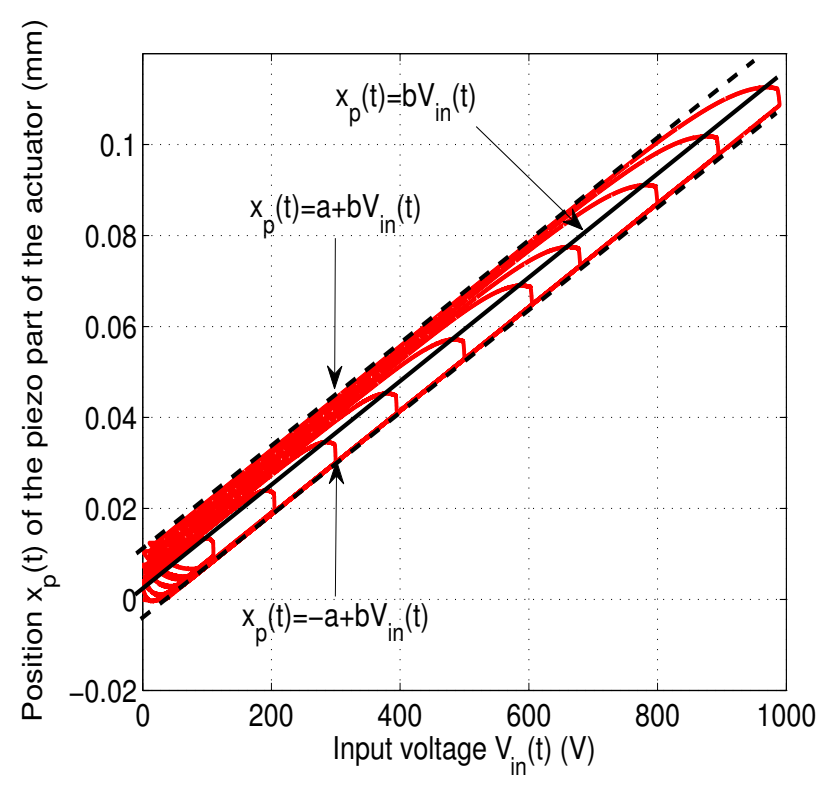

Fig. 7. Hysteresis profile of the PEA: $H\left(x_{p}(t), V_{i n}(t)\right)$

shown in Fig. 8 is regarded, the systems shown in Figs. 6, 7 and 8 can be described by the expressions:

$$
\begin{aligned}
& \frac{M_{p}}{3} \ddot{x}(t)+M_{S K} \ddot{x}_{S K}(t)+K x(t)+D \dot{x}(t)+K_{S K} x_{S K}(t) \\
& +D_{S K} \dot{x}_{S K}(t)+D_{o i l} \dot{x}_{S K}(t)+K_{x}\left(x(t)-\Delta x_{p}\left(V_{i n}(t)\right)\right) \\
& =0
\end{aligned}
$$

where $\Delta x_{p}(t)$ represents the interval function of $x_{p}(t)$ as it is demonstrated in Fig. 7 which, according to eq. (4), can be clearly stated as

$$
\Delta x_{p}(t)=D_{x} \Delta V_{z}(t),
$$

At the end, it is possible to see that eqs. (5) and (7),

$K_{x} \Delta x_{p}(t)=K_{x} D_{x}\left(V_{i n}(t)-R_{0} i(t)-H\left(\Delta x_{p}(t), V_{i n}(t)\right)\right)$,

which indicates the force produced by the PEA. Eq. (6) can be written as follows:

$$
\begin{gathered}
\quad \frac{M_{p}}{3} \ddot{x}(t)+M_{S K} \ddot{x}_{S K}(t)+K x(t)+D \dot{x}(t)+K_{S K} x_{S K}(t) \\
+D_{S K} \dot{x}_{S K}(t)+D_{o i l} \dot{x}_{S K}(t)+K_{x} x(t)=K_{x} \Delta x_{p}\left(V_{i n}(t)\right) .
\end{gathered}
$$


Attention should be paid that in a static and quasi-static conditions it holds:

$$
x_{S K}(t)=i_{w e g} x(t),
$$

with $i_{\text {weg }}$ the position ratio is indicated and it declares that the oil cannot be compressed in the conic oil chamber. $F_{d}(t)$ represents the force acting against the valve because of combustion back pressure inside the cylinder. Considering

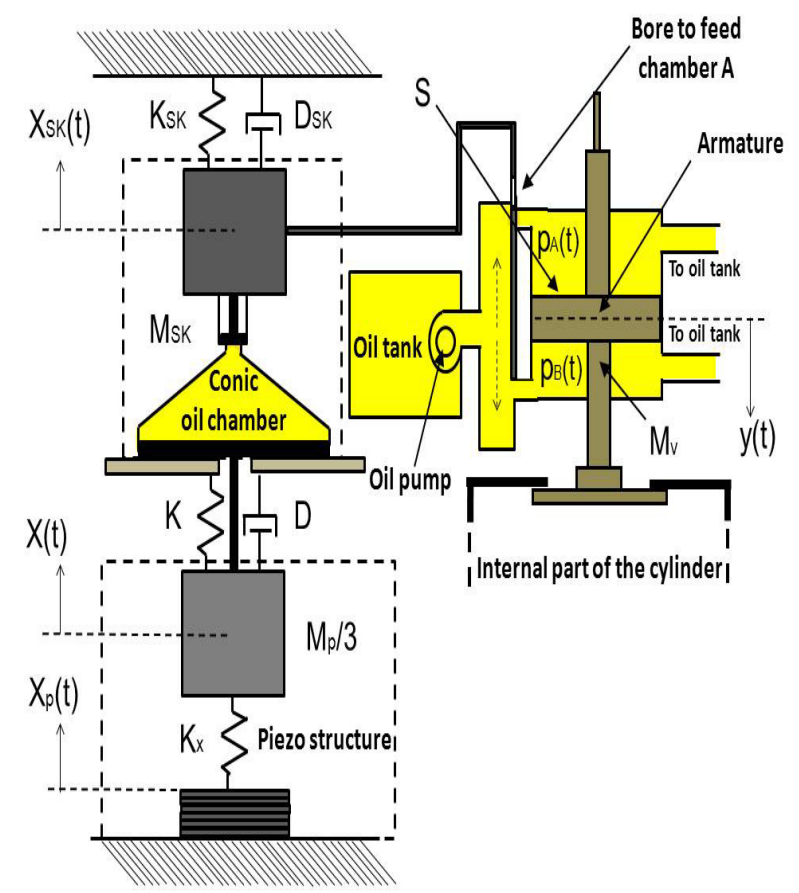

Fig. 8. Mass spring model of the complete actuator

Fig. 7 where an upper bound and a lower bound of the hysteresis curve are pointed out, it can be written that

$$
\Delta x_{p}\left(V_{i n}(t)\right)=\left[\begin{array}{ll}
-a & a
\end{array}\right]+b V_{i n}(t),
$$

$a \in \mathbb{R}$ and $b \in \mathbb{R}$ are two positive constants. Especially,

$$
\triangleq x_{p}\left(V_{i n}(t)\right)=-a+b V_{i n}(t) \text {, }
$$

and

$$
\bar{\Delta} x_{p}\left(V_{i n}(t)\right)=a+b V_{i n}(t) .
$$

After taking this notation into account, the model shown in eq. (6) can be separated into two models:

$$
\begin{aligned}
& a) \\
& \frac{M_{p}}{3} \ddot{x}(t)+M_{S K} \ddot{x}_{S K}(t)+K x(t)+D \dot{x}(t)+K_{S K} x_{S K}(t) \\
& +D_{S K} \dot{x}_{S K}(t)+D_{o i l} \dot{x}_{S K}(t)+K_{x} x(t)=\triangleq x_{p}\left(V_{i n}(t)\right),
\end{aligned}
$$

b)

$\frac{M_{p}}{3} \ddot{x}(t)+M_{S K} \ddot{x}_{S K}(t)+K x(t)+D \dot{x}(t)+K_{S K} x_{S K}(t)$

$+D_{S K} \dot{x}_{S K}(t)+D_{o i l} \dot{x}_{S K}(t)+K_{x} x(t)=\bar{\Delta} x_{p}\left(V_{i n}(t)\right)$,

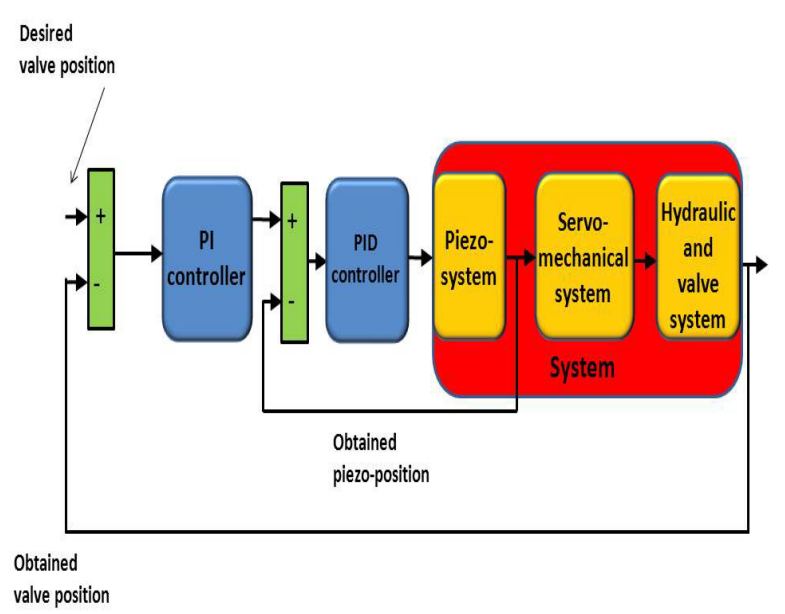

Fig. 9. Proposed control structure

\section{An AdAPTIVE INTERNAL PID CONTROLLER}

The PID control structure shown in this section is quite similar to the sliding control structure presented in [7]. After taking Figs. 6 and 8 into consideration, if the dynamics indicated in (14) and (15) is taken into account in a state space representation, then

$$
\begin{aligned}
\dot{x}_{1}(t)= & x_{2}(t) \\
\dot{x}_{2}(t)= & \frac{-D x_{2}(t)-W\left(D_{S K}+D_{o i l}\right) x_{2}(t)}{\frac{M_{p}}{3}+M_{S K} W}+ \\
& \frac{-\left(K+K_{x}+K_{S K} W\right) x_{1}(t)}{\frac{M_{p}}{3}+M_{S K} W}+ \\
& \frac{3 K_{x} b V_{i n}(t)+(-1)^{q} a}{\frac{M_{p}}{3}+M_{S K} W}
\end{aligned}
$$

where $q=1,2$.. The system demonstrated in equations (16) and (18) can be stated in the following way:

$$
\left[\begin{array}{c}
\dot{x}_{1}(t) \\
\dot{x}_{2}(t)
\end{array}\right]=\mathbf{f}\left(\mathbf{x}(t), H\left(y(t), V_{i n}(t)\right)\right)+\mathbf{B} V_{i n}(t)
$$

where we can assume that $V_{i n}(t)=V_{z}(t)$,

$$
\begin{aligned}
\mathbf{f}(\mathbf{x}(t), H(y(t), & \left.\left.V_{i n}(t)\right)\right)= \\
& {\left[\begin{array}{c}
x_{2}(t) \\
\frac{-D x_{2}(t)-\left(K+K_{x}+K_{S K} W\right) x_{1}(t)}{\frac{M_{p}}{3}+M_{S K} W}
\end{array}\right], }
\end{aligned}
$$

and $\mathbf{B}=\left[\begin{array}{c}0 \\ \frac{3 K_{x} b+(-1)^{q} a}{\frac{M_{p}}{3}+M_{S K} W}\end{array}\right]$. The following PID controller is defined:

$$
K(t)=\mathbf{G}\left(\mathbf{x}_{d}(t)-\mathbf{x}(t)\right),
$$


where $\mathbf{G}=\left[\begin{array}{cc}P_{i} & D_{i}\end{array}\right]$, and $\mathbf{x}_{d}(t)$ indicate the vector of the needed piezo trajectories. Equation (21) will be as follows:

$$
\begin{aligned}
K_{i}(t)= & {\left[\begin{array}{ll}
P_{i} & D_{i}
\end{array}\right]\left[\begin{array}{l}
x_{1 d}(t)-x_{1}(t) \\
x_{2 d}(t)-x_{2}(t)
\end{array}\right]+} \\
& I_{i} \int\left(x_{1 d}(t)-x_{1}(t)\right) d t
\end{aligned}
$$

thus

$$
\begin{array}{r}
K_{i}(t)=P_{i}\left(x_{1 d}(t)-x_{1}(t)\right)+D_{i}\left(x_{2 d}(t)-x_{2}(t)\right)+ \\
I_{i} \int\left(x_{1 d}(t)-x_{1}(t)\right) d t
\end{array}
$$

$P_{i}$ and $D_{i}$ are the internal $\mathrm{P}$ and the intern $\mathrm{D}$ parameters of the PID controller. Considering the Lyapunov function as follows:

$$
V\left(K_{i}\right)=\frac{K_{i}^{2}(t)}{2}
$$

then it follows that:

$$
\dot{V}\left(K_{i}\right)=K_{i}(t) \dot{K}_{i}(t) .
$$

In order to find the stability of the solution $s(t)=0$, the following function can be chosen:

$$
\dot{V}\left(K_{i}\right)=-\eta(t) K_{i}^{2}(t),
$$

with $\eta>0$. After the comparison of (25) with (26), the sequential relationship is obtained:

$$
K_{i}(t) \dot{K}_{i}(t)=-\eta K_{i}^{2}(t)
$$

and at the end

$$
K_{i}(t)\left(\dot{K}_{i}(t)+\eta K_{i}(t)\right)=0 .
$$

The constructive solution can be derived from the condition

$$
\dot{K}_{i}(t)+\eta K_{i}(t)=0 .
$$

From (21) it comes:

$$
\begin{array}{r}
\dot{K}_{i}(t)=\mathbf{G}\left(\dot{\mathbf{x}}_{d}(t)-\dot{\mathbf{x}}(t)\right)+I_{i}\left(x_{1 d}(t)-x_{1}(t)\right)= \\
\mathbf{G}_{d}(t)-\mathbf{G} \dot{\mathbf{x}}(t)+I_{i}\left(x_{1 d}(t)-x_{1}(t)\right) .
\end{array}
$$

The main idea is finding a $u_{e q}(t)$, an equivalent input, and after that a $V_{i n}(t)$, such that $\dot{\mathbf{x}}(t)=\dot{\mathbf{x}}_{d}(t)$. For that, from (19) it comes that:

$$
\dot{\mathbf{x}}(t)=\dot{\mathbf{x}}_{d}(t)=\mathbf{f}\left(x_{d}(t), H\right)+\mathbf{B} V_{i n}(t),
$$

and from (30) the following relationship is got:

$$
\begin{array}{r}
\dot{K}_{i}(t)=\mathbf{G} \dot{\mathbf{x}}_{d}(t)-\mathbf{G f}\left(x_{d}(t), H\right)-\mathbf{G B} V_{i n}(t)+ \\
I_{i}\left(x_{1 d}(t)-x_{1}(t)\right)=\mathbf{G B}\left(u_{e q}(t)-V_{i n}(t)\right)+ \\
I_{i}\left(x_{1 d}(t)-x_{1}(t)\right),
\end{array}
$$

where $u_{e q}(t)$ represents an equivalent input which can be written as follows:

$$
u_{e q}(t)=(\mathbf{G B})^{-1} \mathbf{G}\left(\dot{\mathbf{x}}_{d}(t)-\mathbf{f}\left(x_{d}(t), H\right)\right) .
$$

After putting (32) in (29) the following relationship is got:

$$
\mathbf{G B}\left(u_{e q}(t)-V_{i n}(t)\right)+\eta K_{i}(t)=0,
$$

and in particular

$$
V_{i n}(t)=u_{e q}(t)+(\mathbf{G B})^{-1} \eta K_{i}(t) .
$$

Normally it is not easy to calculate $u_{e q}(t)$. If eq. (32) is written in a discrete form after using Euler approximation, then it comes:

$$
\frac{K_{i}\left((k+1) T_{s}\right)-K_{i}\left(k T_{s}\right)}{T_{s}}=\mathbf{G B}\left(u_{e q}\left(k T_{s}\right)-V_{i n}\left(k T_{s}\right)\right) .
$$

If eq. (35) is written in a discrete form, it follows that:

$$
V_{i n}\left(k T_{s}\right)=u_{e q}\left(k T_{s}\right)+(\mathbf{G B})^{-1} \eta K_{i}\left(k T_{s}\right) .
$$

Eq. (36) can be written as

$u_{e q}\left(k T_{s}\right)=V_{i n}\left(k T_{s}\right)+(\mathbf{G B})^{-1} \frac{K_{i}\left((k+1) T_{s}\right)-K_{i}\left(k T_{s}\right)}{T_{s}}$.

Eq. (38) can be written to one-step backward as follows:

$$
\begin{aligned}
& u_{e q}\left((k-1) T_{s}\right)=V_{\text {in }}\left((k-1) T_{s}\right)+(\mathbf{G B})^{-1} \\
& \frac{K_{i}\left(k T_{s}\right)-K_{i}\left((k-1) T_{s}\right)}{T_{s}} .
\end{aligned}
$$

Function $u_{e q}(t)$ is a continuous one, it can be written

$$
u_{e q}\left(k T_{s}\right) \approx u_{e q}\left((k-1) T_{s}\right) .
$$

Considering equation (40), then equation (39) becomes

$$
\begin{aligned}
& u_{e q}\left(k T_{s}\right)=V_{i n}\left((k-1) T_{s}\right)+(\mathbf{G B})^{-1} \\
& \frac{K_{i}\left(k T_{s}\right)-K_{i}\left((k-1) T_{s}\right)}{T_{s}} .
\end{aligned}
$$

Putting (41) into (37)

$$
\begin{aligned}
& V_{i n}\left(k T_{s}\right)=V_{i n}\left((k-1) T_{s}\right)+(\mathbf{G B})^{-1} \\
& \left(\eta K_{i}\left(k T_{s}\right)+\frac{K_{i}\left(k T_{s}\right)-K_{i}\left((k-1) T_{s}\right)}{T_{s}}\right),
\end{aligned}
$$

and at the end

$$
\begin{aligned}
& V_{i n}\left(k T_{s}\right)=V_{i n}\left((k-1) T_{s}\right)+\left(\mathbf{G B} T_{s}\right)^{-1} \\
& \quad\left(\eta T_{s} K_{i}\left(k T_{s}\right)+K_{i}\left(k T_{s}\right)-K_{i}\left((k-1) T_{s}\right)\right) .
\end{aligned}
$$

Through matrix $\mathbf{B}$ the control law is a switching one

\section{Simulation}

The control law in (43) is implemented and Fig. 10 represents the result about the position of an exhaust valve with $8000 \mathrm{rpm}$. Figure 11 indicates the last result about the velocity regarding the exhaust valve with $8000 \mathrm{rpm}$. Figure 12 indicates the force acting on the piezo part of the suggested actuator. But, the force acting directly on the valve at the opening time has a peak value equal to $700 \mathrm{~N}$ circa. This kind of actuators have this benefit. The sampling time equals $20 \times 10^{-6} \mathrm{~s}$, with respect to the hardware characteristic of the microprocessor which is in use. 


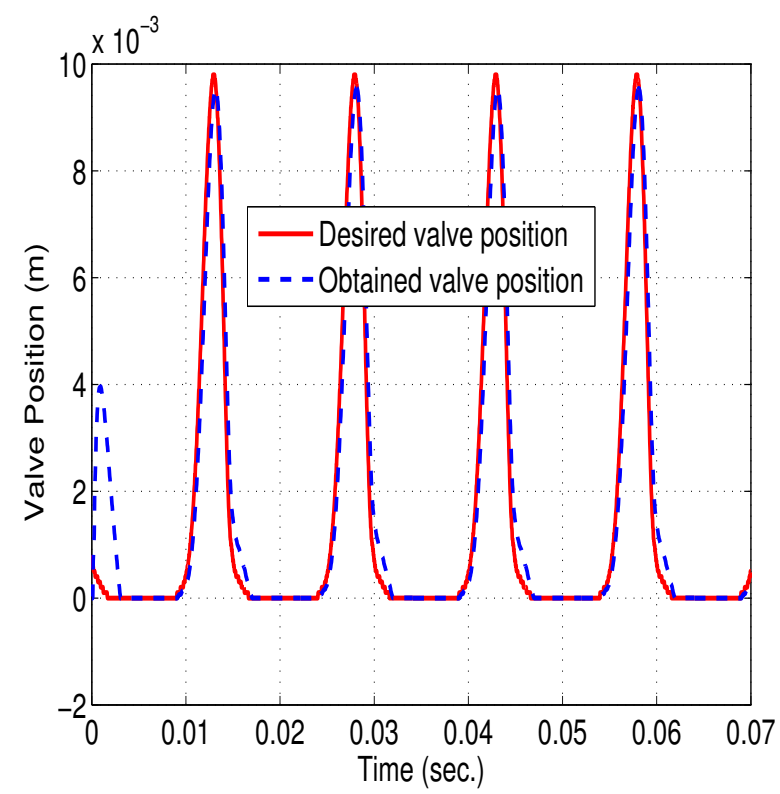

Fig. 10. Desired and obtained valve positions corresponding to $8000 \mathrm{rpm}$

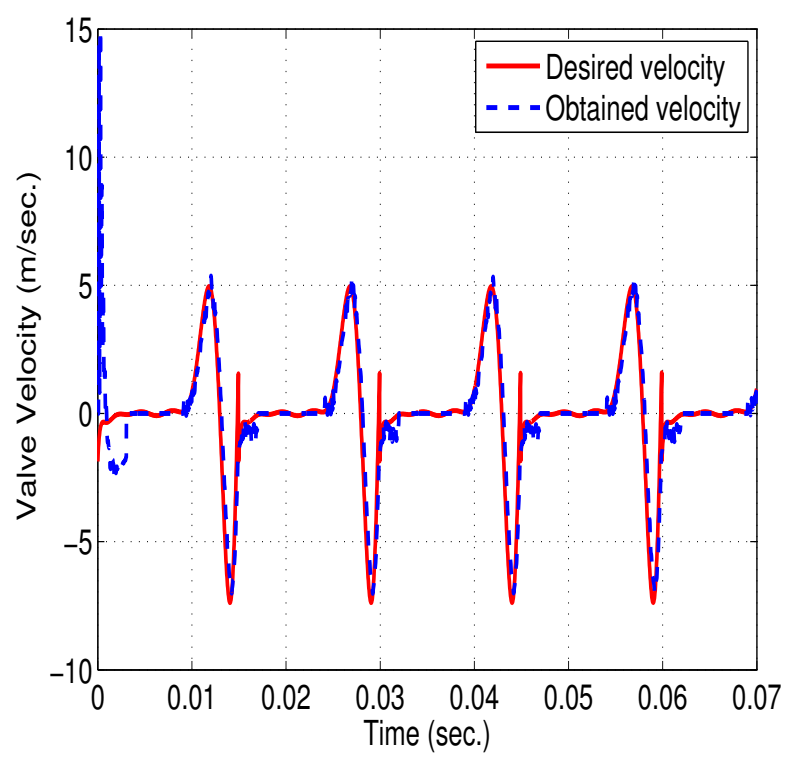

Fig. 11. Desired and obtained valve velocity $(8000 \mathrm{rpm})$

\section{CONCLUSION}

The main topic of this work is a hybrid actuator made of a PEA and a hydraulic part and its control structure for automotive applications. The main scope of this contribution is to show the benefits of both, the precision of the piezo and the force of the hydraulic part. The suggested control scheme is a cascade PI-PID-PI controller. Simulations are shown.

\section{REFERENCES}

[1] P. Mercorelli. A switching kalman filter for sensorless control of a hybride hydraulic piezo actuator using mpc for camless internal

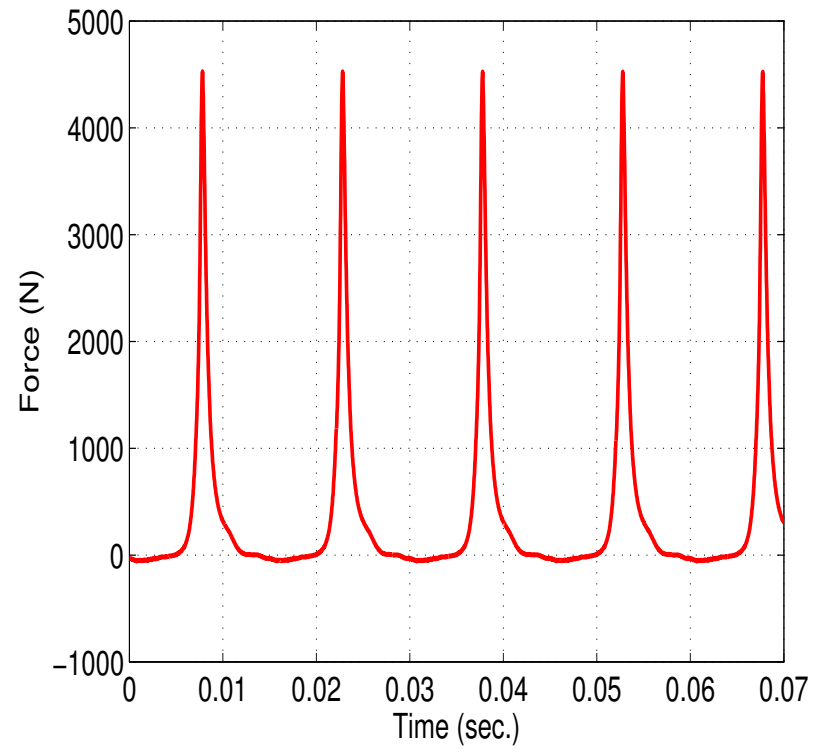

Fig. 12. Force of the internal combustion considering $8000 \mathrm{rpm}$

combustion engine. In 2012 Multi-Conference on Systems and Control, pages 980-985, Dubrovnik, (Croatia), 2012.

[2] P. Mercorelli and Nils Werner. A hybrid hydraulic piezo actuator for camless internal combustion wngines controlled with an mpc and an affine feedforward structure. Procedia Engineering, 50:685-696, 2012.

[3] P. Mercorelli. Robust feedback linearization using an adaptive pd regulator for a sensorless control of a throttle valve. Mechatronics a journal of IFAC. Elsevier publishing, 19(8):1334-1345, 2009.

[4] P. Mercorelli, S. Liu, and K. Lehmann. Robust flatness based control of an electromagnetic linear actuator using adaptive pid controller. In 42nd IEEE Conference on Decision and Control, 2003.

[5] H.J.M.T.A. Adriaens, W. L. de Koning, and R. Banning. Modeling piezoelectric actuators. IEEE/ASME Transactions on Mechatronics, 5(4):331-341, 2000.

[6] Y.-C. Yu and M.-K. Lee. A dynamic nonlinearity model for a piezo-actuated positioning system. In Proceedings of the 2005 IEEE International Conference on Mechatronics, 2005.

[7] J. Lee, D. Lee, and S. Won. Precise tracking control of piezo actuator using sliding mode control with feedforward compensation. In Proceedings of the SICE Annual Conference 2010, 2010.

Creative Commons Attribution License 4.0 (Attribution 4.0 International, CC BY 4.0)

This article is published under the terms of the Creative Commons Attribution License 4.0 https://creativecommons.org/licenses/by/4.0/deed.en_US 